\title{
Prognosis in heart failure with preserved left ventricular systolic function: prospective cohort study
}

Philip A MacCarthy, Mark T Kearney, James Nolan, Amanda J Lee, Robin J Prescott, Ajay M Shah, W Paul Brooksby, Keith A A Fox

Department of Cardiology, Guy's, King's, and

St Thomas's Schoo of Medicine, London SE5 9PJ

Philip A MacCarthy consultant

Mark T Kearney senior lecturer

Ajay M Shah British Heart Foundation professor

North Staffordshire Cardiac Centre, Stoke on Trent, ST4 6QG

James Nolan consultant

Medical Statistics Unit, University of Edinburgh, Edinburgh

EH3 9YW

Amanda J Lee statistician

Robin J Prescott statistician

Pontefract and Wakefield Hospitals, Pontefract WF8 1PL

W Paul Brooksby consultant cardiologist

Department of Cardiology,

University of

Edinburgh

Keith A A Fox

British Heart

Foundation professor

Correspondence to:

M T Kearney

mark.kearney@

kcl.ac.uk

BMJ 2003;327:78-9

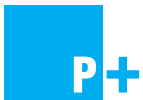

A graph showing survival curves is available on bmi.com
Chronic heart failure due to left ventricular systolic impairment is characterised by a poor prognosis and abnormalities of cardiac structure, autonomic and neurohumoral function, and fluid and electrolyte homoeostasis, all of which are thought to contribute to the pathophysiology of this condition. However, some studies have found that $30-50 \%$ of all patients with chronic heart failure have preserved left ventricular systolic function. ${ }^{1}$ Despite this, the natural course of the condition in these patients is controversial, and their pathophysiological characterisation poor. As a result, optimum treatment strategies are unclear. We looked at five year mortality in patients recruited to a large cohort study of chronic heart failure, comparing those having impairment of left ventricular function with those having preserved function.

\section{Participants, methods, and results}

We have published details of the United Kingdom heart failure evaluation and assessment of risk trial (UK-HEART) previously. ${ }^{2}$ Five hundred and fifty three unselected ambulant patients were prospectively recruited from April 1993 to December 1995. Patients

Characteristics of 522 patients with chronic heart failure and preserved or impaired left ventricular systolic function. Values are means (standard deviations) unless stated otherwise

\begin{tabular}{|c|c|c|c|}
\hline Characteristic & Preserved function $(\mathrm{n}=163)$ & Impaired function ( $\mathrm{n}=359)$ & $P$ value \\
\hline Age & $62.5(10.7)$ & $62.3(9.10)$ & $0.8^{*}$ \\
\hline No $(\%)$ of men & $117(72)$ & $287(80)$ & $0.07 \dagger$ \\
\hline \multicolumn{4}{|l|}{ NYHA class (No (\%)): } \\
\hline 1 & $3(2)$ & 7 (2) & $<0.001 \dagger$ \\
\hline II & $119(73)$ & $190(53)$ & \\
\hline III & $41(25)$ & $162(45)$ & \\
\hline Hypertension (№ (\%)) & $10(6)$ & $11(3)$ & $0.2 \dagger$ \\
\hline Ischaemic heart disease (№ (\%)) & $124(76)$ & $284(79)$ & $0.5 \dagger$ \\
\hline Myocardial infarction (No (\%)) & $106(65)$ & $237(66)$ & $0.9 \dagger$ \\
\hline Angiotensin converting enzyme inhibitor (No (\%)) & $127(78)$ & $294(82)$ & $0.4 \dagger$ \\
\hline Median (interquartile range) frusemide dose (mg) & $40(40$ to 80$)$ & $80(40$ to 80$)$ & $<0.001 \#$ \\
\hline$\beta$ blocker (No (\%)) & $11(7)$ & $22(6)$ & $0.9 \#$ \\
\hline Digoxin (No (\%)) & $23(14)$ & $75(21)$ & $0.09 \#$ \\
\hline Creatinine $(\mu \mathrm{mol} / \mathrm{l})$ & $115(39)$ & $123(42)$ & $<0.05^{*}$ \\
\hline Sodium (mmol/l) & $139.9(2.60)$ & $139.6(3.44)$ & $0.3^{\star}$ \\
\hline Potassium (mmol/l) & $4.36(0.43)$ & $4.33(0.51)$ & $0.4^{\star}$ \\
\hline Plasma glucose $(\mathrm{mmol} / \mathrm{l})$ & $5.90(2.82)$ & $5.92(2.31)$ & $0.9^{*}$ \\
\hline Median (interquartile range) cardiothoracic ratio & $0.50(0.47$ to 0.55$)$ & $0.53(0.50$ to 0.57$)$ & $<0.001 \#$ \\
\hline Left ventricular end systolic diameter $(\mathrm{cm})$ & $4.08(0.96)$ & $5.50(0.98)$ & $<0.001^{*}$ \\
\hline Left ventricular end diastolic diameter $(\mathrm{cm})$ & $5.79(1.01)$ & $6.43(0.95)$ & $<0.001^{*}$ \\
\hline Left ventricular hypertrophy (№ (\%)) & $20(12)$ & $36(10)$ & $0.7 \dagger$ \\
\hline Median (interquartile range) heart rate (beats/min) & 69 (63 to 82$)$ & 76 (66 to 89 ) & $<0.001 \#$ \\
\hline Non-sustained ventricular tachycardia (№ (\%)) & $41(25)$ & $158(44)$ & $<0.001 \dagger$ \\
\hline Median (interquartile range) SDNN (ms) & 115 (91 to 144$)$ & 110 (81 to 142$)$ & $0.1 \#$ \\
\hline Total mortality at five years (№ (\%)) & $41(25)$ & $151(42)$ & $<0.001 \dagger$ \\
\hline
\end{tabular}

NYHA=New York Heart Association.

SDNN=standard deviation of all normal $R-R$ intervals over 24 hours.

Left ventricular hypertrophy was determined on the basis of voltage criteria.

${ }^{*}$ Student's $t$ test (for normally distributed data).

$+\chi^{2}$ test (for categorical variables).

\#Mann-Whitney U test (for non-normally distributed data). were enrolled if they had had stable, symptomatic chronic heart failure for at least three months (other primary causes of symptoms were excluded). As well as symptoms of chronic heart failure, all patients had evidence of cardiac dysfunction documented at the index assessment by one or more of the following: systolic left ventricular dysfunction on echocardiography or radionuclide ventriculography; cardiothoracic ratio $>0.55$; and pulmonary venous congestion and/or upper lobe venous diversion on chest radiography. Left ventricular hypertrophy was assessed from electrocardiography (on the basis of Sokolow-Lyon criteria). All patients had 24 hour ambulatory monitoring for arrhythmia analysis and assessment of heart rate variability. A global index of total heart rate variability - the standard deviation of all normal R-R intervals (SDNN) (with a low value indicating a disadvantageous neurohumoral profile)-was derived from this recording. All patients were logged on the NHS Central Register (part of the Office of Population Censuses and Surveys), which notified the investigators when patients died.

Most studies confirm that patients with an ejection fraction $\geq 50 \%$ can be considered to have preserved left ventricular systolic function. We therefore chose this 
value to dichotomise patients into those with preserved $(\geq 50 \%)$ and those with impaired $(<50 \%)$ function. ${ }^{4}$

In all, 522 patients had adequate measurements of ejection fraction, of whom $163(31 \%)$ had values $\geq 50 \%$ and $359(69 \%)<50 \%$. Information on deaths was recorded to April 2000, allowing five year survival status to be determined for all patients. The table shows the characteristics of the two groups and statistical methods.

Five year mortality was substantial in both groups but significantly greater in patients with impaired left ventricular systolic function $(41.5 \%$ v 25.2\%, P < 0.001$)$. Twenty five per cent of patients with preserved function had non-sustained ventricular tachycardia. Both groups had similar SDNN measurements, which were lower than previously shown in age matched healthy control subjects.

\section{Comment}

Mortality is significantly greater in patients with chronic heart failure and impaired left ventricular systolic function than in those with preserved systolic function. However, even the patients with preserved systolic function have a $25 \%$ five year mortality. Therefore, clinical heart failure itself has a poor long term prognosis, irrespective of electrocardiographically determined left ventricular systolic function. Autonomic function was abnormal in both groups, and this, allied to the presence of non-sustained ventricular tachycardia and left ventricular hypertrophy, may contribute to the high mortality found in the patients with preserved systolic function.

Our findings add to those of a recent study by Kitzman et al, who found that patients with chronic heart failure and preserved left ventricular systolic function have similar, but not as severe, pathophysiological derangements to those with impaired systolic function. ${ }^{4}$ These and our data suggest that established treatments for systolic heart failure may also have a role in patients with chronic heart failure and preserved left ventricular systolic function.

Contributors: PAM and MTK conceived the original idea, did the retrieval, analysis, and interpretation of the data, and wrote the paper. MTK helped to collect the raw data. KAAF, JN, and WPB were responsible for the original concept of the UK-HEART study, designed the database, and collected the data on patients; they also reviewed and revised the current paper. AJL and RJP helped with data acquisition and advised on statistical analysis. AJL did a large part of the statistical analysis. AMS helped with the conception of this project, the study design, the interpretation of data, and the revision of the manuscript. MTK is the guarantor for the paper.

Funding: PAM was the recipient of an advanced training scholarship from the British Heart Foundation; MTK is a British Heart Foundation (BHF) intermediate research fellow; and AMS and KAAF hold BHF chairs in cardiology.

Competing interests: None declared.

Ethical approval: Local ethics committees at each participating hospital approved the protocol, and informed written consent was obtained from all patients.

Vasan RS, Benjamin EJ, Levy D. Prevalence, clinical features and prognosis of diastolic heart failure: an epidemiologic perspective. I Am Coll sis of diastolic heart fail

2 Nolan J, Batin PD, Andrews R, Lindsay SJ, Brooksby P, Mullen M, et al. Prospective study of heart rate variability and mortality in chronic heart failure. Results of the United Kingdom heart failure evaluation and assessment of risk trial (UK-Heart). Circulation 1998;98:1510-6.

3 Kearney MT, Fox KA, Lee AJ, Prescott RJ, Shah AM, Batin PD, et al. Predicting death due to progressive heart failure in patients with mild-tomoderate chronic heart failure. J Am Coll Cardiol. 2002;40:1801-8.

4 Kitzman DW, Little WC, Brubaker PH, Anderson RT, Hundley WG, Marburger CT, burger CI, et al. Pathophysiological characterization of isolated diastolic

Bigger JT Jr, Fleiss JL, Steinman RC, Rolnitzky LM, Schneider WJ, Stein PK. RR variability in healthy, middle-aged persons compared with patients with chronic coronary heart disease or recent acute myocardial infarction. Circulation 1995;9:1936-43.

(Accepted 15 April 2003)

\title{
Should same anaesthetist do preoperative anaesthetic visit and give subsequent anaesthetic? Questionnaire survey of anaesthetists
}

\author{
Bruno Simini, Guido Bertolini for the GiViTI group (Gruppo italiano per la Valutazione degli \\ interventi in Terapia Intensiva)
}

The preoperative anaesthetic visit is done to assess the patient's fitness for surgery, to discuss the most appropriate anaesthetic technique, to reassure the patient, to obtain informed consent, and to prescribe premedicant drugs. Patients used to be visited by the doctor who later anaesthetised them, ${ }^{1}$ but the preoperative visit and the subsequent anaesthetic are now seldom done by the same anaesthetist. ${ }^{2}$ Patients would rather be anaesthetised by the doctor who saw them, ${ }^{3}$ but anaesthetists' opinions are unexplored.

\section{Participants, methods, and results}

In June 2002 we sent a questionnaire containing two scenarios to anaesthetists belonging to the Gruppo italiano per la Valutazione degli interventi in Terapia Intensiva (GiViTI), a research network of Italian anaesthesia and intensive care units founded in 1991 to promote research and improve clinical practice. In scenario A, "one patient, one anaesthetist," patients are anaesthetised by the anaesthetist who visited them. In scenario B, "one patient, two anaesthetists," one anaesthetist visits a patient and another physician administers the anaesthetic. We asked anaesthetists which scenario is used in their institution, which one they preferred, and to pick from a list (drawn up by a panel of senior anaesthetists, see box) at least one reason for their choice. Assuming 50\% preferences for both choices (worst scenario for estimating sample size), we needed 170 respondents to give a width of $15 \%$ for the
Ospedale Generale Lucca, Italy Bruno Simini consultant anaesthetist

Laboratorio di Epidemiologia Clinica, Istituto di Ricerche Farmacologiche "Mario Negri," 24020 Ranica (Bergamo), Italy Guido Bertolini senior epidemiologist Correspondence to: B Simini bruno.simini@ virgilio.it

BMJ 2003;327:79-80 